\title{
Article \\ Brown-Field Migration Aware Routing and Spectrum Assignment in Backbone Optical Networks
}

\author{
Xiaosong Yu ${ }^{1} \mathbb{D}$, Jiye Wang ${ }^{2}$, Kaixin Zhang ${ }^{1}$, Jiaqi Lv $^{1}$, Yongli Zhao ${ }^{1, * \mathbb{D}}$, Kaiqiang Gao ${ }^{2}$, Xuerang Guo ${ }^{3}$ \\ and Jie Zhang ${ }^{1}$
}

check for

updates

Citation: Yu, X.; Wang, J.; Zhang, K.; Lv, J.; Zhao, Y.; Gao, K.; Guo, X.; Zhang, J. Brown-Field Migration Aware Routing and Spectrum Assignment in Backbone Optical Networks. Appl. Sci. 2022, 12, 438 https://doi.org/10.3390/ app12010438

Academic Editor: Mirosław Klinkowski

Received: 7 November 2021 Accepted: 30 December 2021 Published: 3 January 2022

Publisher's Note: MDPI stays neutral with regard to jurisdictional claims in published maps and institutional affiliations.

Copyright: (C) 2022 by the authors. Licensee MDPI, Basel, Switzerland. This article is an open access article distributed under the terms and conditions of the Creative Commons Attribution (CC BY) license (https:// creativecommons.org/licenses/by/ $4.0 /)$.
1 School of Electronic Engineering, Beijing University of Posts and Telecommunications, Beijing 100876, China; xiaosongyu@bupt.edu.cn (X.Y.); zhangkaixin@bupt.edu.cn (K.Z.); lv_jiaqi@bupt.edu.cn (J.L.); lgr24@bupt.edu.cn (J.Z.)

2 China Electric Power Research Institute, Beijing 100192, China; wangjiye@epri.sgcc.com.cn (J.W.); xintong-gaokaiqiang@epri.sgcc.com.cn (K.G.)

3 Information and Communication Technology Center, State Grid Xinjiang Electric Power Research Institute, Urumqi 830011, China; lululu@bupt.edu.cn

* Correspondence: yonglizhao@bupt.edu.cn

\begin{abstract}
With the development of optical networks technology, broad attention has been paid to flexible grid technology in optical networks due to its ability to carry large-capacity information as well as provide flexible and fine-grained services through on-demand spectrum resource allocation. However, a one-time green-field deployment of a flexible grid network may not be practical. The transition technology called the fixed/flex-grid optical networks is more applicable and highly pragmatic. In such network, many nodes would likely be upgraded from a fixed-grid to flex-grid. In fact, dynamic service provisioning during the process of a node upgrade in fixed/flex-grid optical networks have become a challenge because the service connection can be easily interrupted, which leads to considerable data loss because of node upgrade. To overcome this challenge, we propose a brown-field migration aware routing and spectrum assignment (BMA-RSA) algorithm in fixed/flex-grid optical networks. The aim is to construct a probabilistic migration label $(P M L)$ model. The welldesigned label setting of $P M L$ can balance the relationship between distance and node-upgrade probability. Dynamic service provisioning operations are undertaken based on the PML model to achieve a migration-aware dynamic connection before network migration occurs. We also evaluate the performance of different service provisioning strategies under different traffic models. The simulation results show that the BMA-RSA algorithm can achieve: (1) the tradeoff between distance and node upgrade probability during the process of service provisioning; (2) lower service interruption compared with the traditional non-migration aware K-shortest-path routing and spectrum assignment algorithm.
\end{abstract}

Keywords: node upgrade; probabilistic migration label; traffic model; migration aware service provisioning

\section{Introduction}

With the expansion of network scale and the continuous emergence of high-traffic services, such as data center services and cloud-based services [1-3], explosive traffic carried by the optical networks has led to an urgent demand for bandwidth resources. Currently, researchers are exploring more advanced optical transmission and networking technologies to improve network capacity [4-6]. In this case, flexible grid technology has attracted extensive attention due to its fine-grained, high flexibility and efficient network resource-utilization characteristics [7,8]. Compared with the fixed-grid wavelength division multiplexing (WDM) optical networks, the emerging optical networks architecture, which is based on flexible-grid technology, can overcome the limitation of rigid capacity for optical channel and provide efficient on-demand spectrum allocation with fine 
granularity spectrum slots, such as $12.5 \mathrm{GHz}$ [9], which can greatly improve spectrum resource utilization $[10,11]$. Additionally, new types of optical networks components, such as, flex-grid optical cross-connects built on Liquid Crystal on Silicon-based wavelength selective switches (WSSs) [12] and Sliceable Bandwidth-Variable Transponders [13] have been introduced to optical networks to support flexible grid technology, which greatly foster the development of novel network architecture.

The flex-grid optical networks use technologies such as optical cross-connects with variable bandwidth, wavelength selective switches, and sliceable bandwidth transponders to transmit network data. Flex-grid optical networks abandon the wavelength constraints, and provide more flexible and fine-grained services through on-demand spectrum resources allocation and the adaptive selection of modulation formats compared with fixed-grid optical networks. Super-channels [14] can be established between flex-grid nodes to achieve ultra-high-speed optical channel transmission, which improves spectrum efficiency and network transmission capacity. However, due to technical cost and feasibility constraints, it is unrealistic to complete the upgrade deployment of the entire network at one time. The transition technology called fixed/flex-grid optical networks where both fixed-and flex-grid nodes co-exist is more practical before complete migration to flex-grid network. Through the deployment of the flexible network node, the capacity of a network can be increased, while preserving the already made investment. The fixed/flex-grid optical networks presents new challenges and limitations at the network level, since the spectrum is shared as a common resource. Different from the fixed-grid ROADMs, for which the central frequencies and spectrum grids are defined as 50 or $100 \mathrm{GHz}$, the flex-grid ROADMs have finer granularity, e.g., 6.25 or $12.5 \mathrm{GHz}$. Figure 1 illustrates four cases of spectrum allocation in fixed/flex-grid optical networks scenario. The slot-width of fixed-grid node and flex-grid node are defined as $50 \mathrm{GHz}$ and the multiple of $12.5 \mathrm{GHz}$, respectively. In Figure 1a, a light path request of $100 \mathrm{~Gb}$ /s between two fixed-gird nodes traverses another fixed-grid node. Due to the characteristics of the fixed-grid nodes, a $50 \mathrm{GHz}$ channel which represents one fixed-grid slot is needed on both of the links to carry the $100 \mathrm{~Gb} / \mathrm{s}$ request. In Figure 1b, for the request carried by the second link, only three flex-grid slots are sufficient because the established super optical channel between two flex-grid nodes has finer granularity. For the $200 \mathrm{~Gb} / \mathrm{s}$ request in Figure 1c, only $75 \mathrm{GHz}$ which represents six flex-grid slots is required on both links. In Figure 1d, to carry the $40 \mathrm{~Gb} / \mathrm{s}$ request, two flex-grid slots are required for the first link and one fixed-grid slot is required for the following fixed-grid link.

(a)

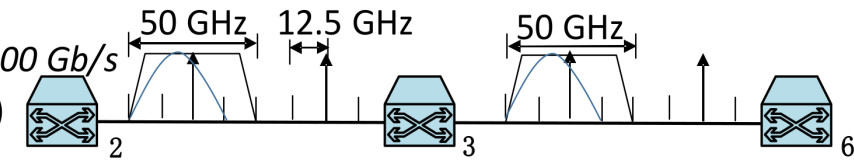

(b)

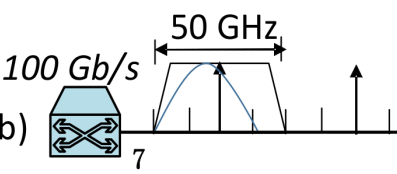

(c)

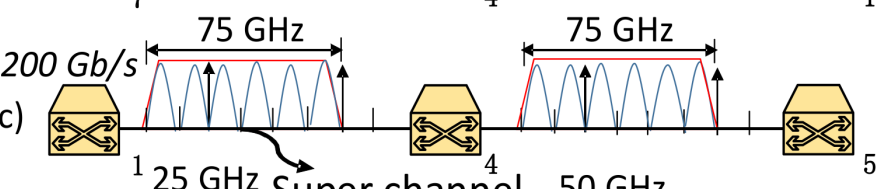

(d)

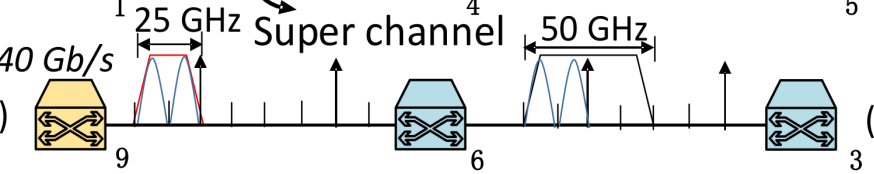

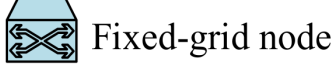

Flex-grid node

e)

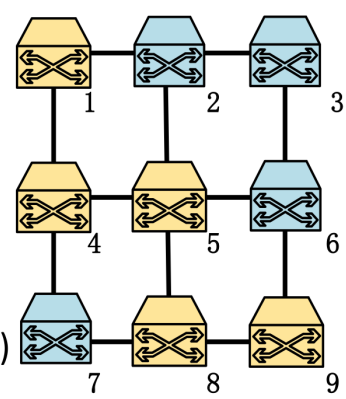

Figure 1. Different mixed-grid scenarios. (a) The 1st case (b) The 2nd case (c) The 3rd case (d) The 4 th case (e) 9-node topology. 
With the development of technology, an elastic optical network has been widely used because it can break the limitation of the rigid capacity for optical channel and provide efficient on-demand spectrum allocation with fine granularity spectrum slots. Most of the equipment that has been deployed is based on fixed-grid, on and in order to improve the utilization of spectrum resources, some nodes will be selected to support flex-grid technology in the future. However, the decision as to which nodes will be selected for upgrade, and the number of nodes selected for upgrade, depends on a number of factors such as network bottleneck, network topology, traffic profile, economic cost, network operator's policy and so on. Consequently, in such optical networks with potential upgrade nodes, how to avoid service interruption on the path is a significant problem to study.

In this study, on the basis of deployed fixed-grid optical networks with potential upgrade nodes, in order to avoid service connection interruption and traffic loss caused by node upgrade and keep stable and reliable service supply at the same time, we identify how to provide migration-aware dynamic connection provisioning, when the nodes in fixed/flexgrid optical networks gradually upgrade from fixed-grid to flex-grid. We evaluate the performance through a simulation and analyze the simulation results compared with the baseline algorithm in terms of the bandwidth-blocking ratio and connection-interruption ratio under uniform and non-uniform traffic models. The major contributions of this work are two-fold: (1) we describe the probabilistic migration label model $(P M L)$ to establish the relationship between link distance and node upgrade (2) and we devise a brown-field migration-aware routing and spectrum assignment (BMA-RSA) algorithm to provide migrationaware dynamic service provisioning upon service arrival. The rest of paper is organized as follows. Section II briefly introduces the problem of service provisioning during the process of node upgrade in fixed/flex-grid optical networks. In section III, we give the probabilistic migration label model to describe the relationship between distance and node upgrade. Then, we elaborate the proposed brown-field migration aware routing and spectrum assignment $(B M A-R S A)$ algorithm. Section IV includes the simulation results and detailed analysis of the proposed algorithm and other algorithm. Finally, section V concludes the work.

\section{Related Work}

Based on the consideration of CAPEX and OPEX [15], a one-time deployment of flex-grid technology for the entire network may not be economically viable. Consequently, the brown-field deployment network, as the basis of the existing fixed-grid network in which both fixed-grid and flex-grid technologies co-exist with seamless interoperability, is a more realistic scenario [16,17]. In fixed/flex-grid optical networks, many technologies have been applied to support communication between WDM nodes and elastic optical network (EON) nodes, such as the virtual concatenation technique [18]. At the same time, to address the problem of spectral path allocation, dynamic resource allocation algorithm with mixed grid recognition considering the interoperability limitations was designed and reported in [19]. The gradual evolution from fixed-grid networks to flex-grid networks, called migration, has been widely investigated [20-25]. Authors in [20,21] studied various migration options from fixed-grid to flex-grid and different impacts on flexibility. With regard to the planning of physical layer constraints from fixed-grid to flex-grid optical networks, details are available in [22]. In [23], the author proposed an energy-efficient traffic grooming scheme to solve the problem of high energy consumption in fixed/flexgrid optical networks. In our previous investigations [24-27], the problem of how to select fixed-grid nodes existing in network upgrade to flex-nodes and related routing strategies have been studied.

When the process of network migration begins (either by upgrading the fully reconfigurable optical add-drop multiplexer (ROADMs) [25], or by upgrading the WSSs [26]), the existing services in the network will be interrupted, causing a large amount of traffic-loss since the fiber capacity is extraordinarily huge. In such fixed/flex-grid optical networks, to establish stable connections for services, various traditional protection techniques have been extended in optical migration scenario [28]. By applying the method of establishing a 
pair of working and protection paths for the service, the problem of connection interruption can be greatly improved, such as choosing a node disjoint pair of paths. One of the paths is the primary path for data transmission, and the other is the backup path, similar to Dedicated Path Protection (DDP) scheme [29]. When migration begins, the service carried on the primary path is interrupted, but the service can be quickly restored by switching the primary path to the reserve backup path. Meanwhile, as a path-focused survival mechanism, Shared Backup Path Protection (SBPP) which protect the services through working and backup routes has been optimized in [30,31] for better service protection performance and effective resource sharing. Nevertheless, the strategies directly derived from the traditional service protection strategies need to reserve spectrum resources for the working and protection path for data transmission which will introduce a certain amount of waste of network resources. Meanwhile, previous studies are unaware of node upgrade during the process of service provisioning. If we can take the issue of node upgrade into consideration during the process of service provisioning before the network migration happens, the traffic interruption and data loss can be effectively avoided.

\section{Problem Statement}

In fixed/flex-grid optical networks, as the fixed-grid nodes own different upgrade necessities, in the process of data transmission, the fixed-grid nodes may be upgraded to flex-grid nodes which we called migration. When such migration happens, the traffic load going through the nodes which are selected to be upgraded in network will be affected, even if be interrupted. Since the fiber capacity is considerable, the interruption of the data transmission will lead to significant data loss. Consequently, migration-aware dynamic connection provisioning occurring before network migration has become a new challenge. The following inputs are given to the algorithm. A physical topology $G(V, E, P)$ denotes the network, which consists of a set of optical nodes $V\left(V_{1}, V_{2}, \cdots, V_{n}\right)$, including $V_{F I}$ and $V_{F L}$, represent the set of fixed-grid nodes and flex-grid nodes and optical links link $(i, j)$ corresponds to the physical link from the $i_{t h}$ to the $j_{t h}$ node. $P\left(P_{1}, P_{2}, \cdots, P_{n}\right)$ represents the set of upgrade probabilities that fixed-grid nodes being upgraded to flex-grid nodes. $R(S, D, B)$ represents a set of service connection requests from the source $s$ node to the destination node $d$. $B$ stands for the request bandwidth requirements, including the guard bands.

There is a fixed/flex-grid optical network, including $V_{F I}$ and $V_{F L}$ as illustrated in Figure 2. The probabilities $P$ of a node upgrade from fixed-grid to flex-grid are set as shown in the table. By adjusting the weight coefficient between dis and $\mathrm{P}$, different routing calculation results can be obtained for the service connection request from node 3 to node 12 . If distance is the only consideration, the route 3-7-9-12 can be obtained (which is actually the shortest path routing). However, this route does not consider P of node 7 to be as high as $90 \%$. Later, when network migration occurs, this connection will be interrupted. If the same weight is given to the distance and node upgrade probability, we can obtain another route as 3-2-6-11-12, which avoids connection interruption caused by upgrading node 7. However, if an additional node (i.e., node 6) is scheduled to upgrade, service provisioning will also be interrupted. If more weight is given to node upgrade probability, path 3-4-5-8-10-13-12 is obtained, which can effectively avoid service-provision interruption during the process of network migration. 


\begin{tabular}{|c|c|c|c|c|c|c|c|c|c|c|c|c|c|c|c|c|c|c|c|c|c|c|c|c|}
\hline Node & 1 & 2 & 3 & 4 & 5 & 6 & 7 & 8 & 9 & 10 & 11 & 12 & 13 & 14 & 15 & 16 & 17 & 18 & 19 & 20 & 21 & 22 & 23 & 24 \\
\hline $\begin{array}{c}\text { Upgrading } \\
\text { Probability }\end{array}$ & 0.2 & 0.1 & 0.3 & 0.1 & 0.1 & 0.8 & 0.9 & - & 0.2 & 0.1 & 0.1 & 0.1 & 0.1 & - & - & 0.1 & - & 0.1 & - & 0.2 & 0.1 & 0.1 & 0.1 & 0.1 \\
\hline
\end{tabular}
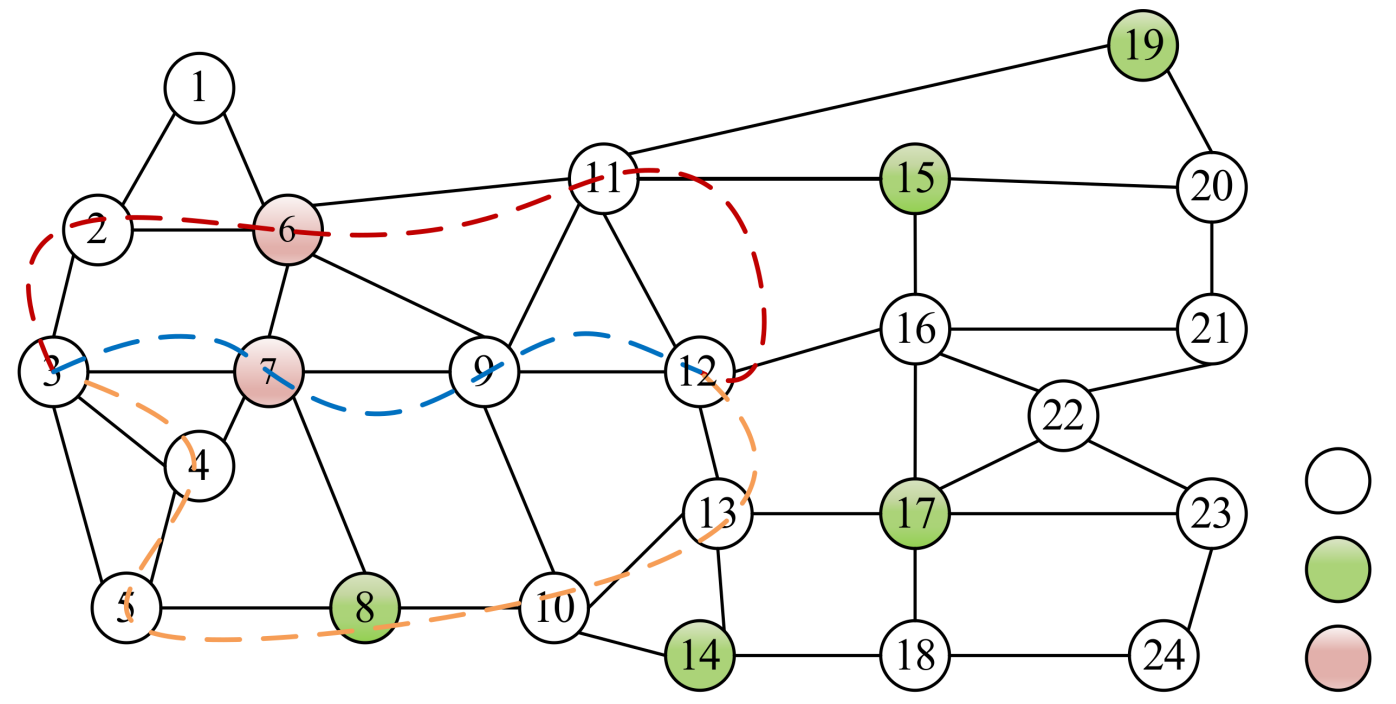

Fixed grid node

Fexible grid node

Potential upgrade node

Figure 2. Migration scenario with traffic survivability issue when two nodes will be selected to be upgraded to flex-grid.

\section{Brown-Field Migration Aware Routing and Spectrum Assignment Algorithm}

In this paper, we design a probabilistic migration label $(P M L)$ model and propose a brown-field migration aware routing and spectrum assignment (BMA-RSA) algorithm to provide migration aware dynamic connection provisioning before the migration begins.

\subsection{Subsection Probabilistic Migration Label Model}

On the basis of the fixed-grid optical networks deployed, in order to increase network flexibility and meet the needs of service carrying, some nodes will be selected as upgrade nodes. Since the traffic distribution and topology of the optical network are different, the probability of upgrading fixed-grid nodes to flex-grid nodes in the network is also different. The upgrade probability of each node is different, due to their various traffic load, degree, economic factors and so on. The different upgrade nodes have noticeable differences in performance. The value of node upgrade probability contains two aspects, one is the self-influence of a node which represents the impact of traffic load. The other is the inter-influence of the nodes, which is closely related with the connectivity. Considering the interaction between nodes, the probabilities of different nodes can affect each other, especially in the case of adjacent nodes because the high bandwidth and spectrum-efficient channel is embodied only between two adjacent flex-grid nodes. Different calculation strategies of node upgrade probability can be obtained by adjusting the weight coefficient of the intra-influence and the inter-influence of the node, based which the node upgrade probability can be calculated [32]. In this paper, we introduced the concept of upgrade probability to describe the importance of upgrading each node in the network to support flex-grid technology, and proposed a migration-aware routing and spectrum allocation algorithm in such a scenario.

Suppose that there are $\mathrm{N}$ nodes in the network, where $p_{i}$ is defined as the upgrade probability of the fixed-grid node $i$, which is calculated by taking self-influence and interinfluence into consideration. The basic of the proposed brown-field migration aware routing and spectrum assignment (BMA-RSA) algorithm is the process of labeling-setting. The label of each node is the value derived from the probability of upgrade and distance from source to the current node. The definition of Equation (1) includes $d i_{s r c, j}$ and $p_{j}$, where $\alpha$ and $\beta$ are 
used as the influenced parameter of the distance and the upgrade probability, respectively. Note that $d i s_{s r c, j}$ is a normalized value, which is denoted by the distance between the source node to the current node over the distance of the longest link in the network topology.

$$
\text { Label }\{j\}=\alpha \times \operatorname{dis}_{s r c, j}+\beta \times p_{j}
$$

During the process of label-setting, a NodeSet maintained by $B M A-R S A$ exists. Initially, only the source node is included in the NodeSet, and on each iteration, $B M A-R S A$ traverses all the nodes outside the NodeSet, selects and adds the node which has the lowest label value to the NodeSet. As shown in Equation (2), the label of the adjacent node is upgraded if the value of the label is reduced because of the expansion of the path from the newly assigned node to those nodes. Note that $d i s_{j, k}$ is also a normalized value.

$$
\text { Label }\{k\}=\operatorname{Label}\{j\}+\alpha \times \text { dis }_{j, k}+\beta \times p_{k}
$$

\subsection{Brown-Field Migration Aware Routing and Spectrum Assignment Algorithm}

Based on the constructed probabilistic migration label model, we proposed a $P M L$ based $B M A-R S A$ algorithm to provide migration-aware dynamic connection provisioning before network migration begins in optical networks, evolving from fixed-grid to flexgrid, which can greatly reduce the interruption of services. By adopting this algorithm in the node upgrade optical networks, the data transmission interruption caused by node upgrade can be effectively avoided in the beginning stages, and traffic survivability can be guaranteed. The related network definitions and algorithm parameters as the basis of the algorithm are explained in Table 1, and the detailed procedures of the proposed PML-based $B M A-R S A$ algorithm are shown in Algorithm 1.

\begin{tabular}{|c|c|}
\hline Notations & Definition \\
\hline$R(S, D, B)$ & A set of service connection requests \\
\hline$G(V, E, P)$ & Physical topology \\
\hline$V_{F I}$ & Set of fixed-grid nodes \\
\hline$V_{F L}$ & Set of flex-grid nodes \\
\hline$S_{F I}$ & Number of slots required in a fixed-grid link for $B$ \\
\hline$S_{F L}$ & Number of slots required in a flex-grid link for $B$ \\
\hline$P_{R}$ & The optimal path for service provisioning \\
\hline$\varnothing_{s}$ & $\begin{array}{l}\text { Boolean value which defines if the source node is a fixed-grid node, the } \\
\text { value is equal to } 0 \text {, otherwise the value is equal to } 1\end{array}$ \\
\hline$\varnothing_{s(l)}$ & $\begin{array}{l}\text { Boolean value which defines if the start node of link } l \text { is a fixed-grid node, } \\
\text { the value is equal to } 0 \text {, otherwise the value is equal to } 1\end{array}$ \\
\hline$\varnothing_{e(l)}$ & $\begin{array}{l}\text { Boolean value which defines if the end node of link } l \text { is fixed-grid node, the } \\
\text { value is equal to } 0 \text {, otherwise the value is equal to } 1\end{array}$ \\
\hline$F_{\text {min }}^{n, i t h}$ & $\begin{array}{l}\text { The minimum unoccupied frequency slot sequence number } i \text { on the } n \text {th } \\
\text { wavelength }\end{array}$ \\
\hline$F^{n, j t h}$ & $\begin{array}{l}\text { The unoccupied frequency slot sequence number } j \text { on the } n \text {th wavelength } \\
\text { for } R\end{array}$ \\
\hline
\end{tabular}

Table 1. Related parameters of $B M A-R S A$ algorithm.

When a connection request $R(S, D, B)$ arrives, firstly, we calculate the node upgrade probability after considering the self-influence and the inter-fluence. According to the upgrade probability of each node and the distance between the source node and the current node, we construct the PML model, which consists of the label of each node. Initially, the NodeSet only contains the source node. As shown in Table 1, we should ideally traverse all nodes that are connected to the source node by links. If a node $j$ has the minimum label, we add the node $j$ into NodeSet and put $\operatorname{link}(s r c, j)$ into $\operatorname{LinkSet}$. For each $\operatorname{link}(i, j)$ for which node $j$ is outside the NodeSet, we find the node that has the minimum value of label and incorporate this node into the NodeSet, and put $\operatorname{link}(i, j)$ into LinkSet. In the process of selecting nodes to join the NodeSet, some nodes already exist in the NodeSet, 
and new nodes need to be integrated into the NodeSet. If the label value of the nodes of choice have a label value higher than the sum of the label values of the nodes that have been added to the NodeSet, then the label of the node needs to be upgraded according to Equation (2). If all nodes are traversed, we will find the optimal path according to the source node and the destination node. The next step is to allocate resources for the path, for which the constraints of spectrum continuity and contiguity are considered. For the nodes on the path, we check and clarify the attributes of each node according to $V_{F I}$ and $V_{F L}$. In the first case, the source node of path $P_{R}$ is a fixed-grid node, so we traverse $S_{F I}$ the consecutive frequency slots starting with $F_{\text {min }}^{n, i t h}$ for each link and check whether they are unoccupied, and if $S_{F I}$ consecutive frequency slots are unoccupied, then allocate spectrum resource and return $S_{F I}, F^{n, j t h}$. Another case is that the source node of path $P_{R}$ is a flex-grid node, and if the start node and the end node of each link $l$ are all flex-grid, we traverse $S_{F L}$ consecutive frequency slots starting with $F_{\text {min }}^{n, i t h}$ then check whether they are unoccupied. If $S_{F L}$ consecutive frequency slots are unoccupied, we allocate spectrum resources for $R$, then return $S_{F L}$ and $F^{n, j t h}$. If there are fixed-grid nodes in the link, we traverse $S_{F I}$ consecutive frequency slots starting with $F_{\text {min }}^{n, i t h}$ and check if they are unoccupied, if so, allocate spectrum resource for $R$, after that return $S_{F I}$ and $F^{n, j t h}$. Otherwise, the process of service provisioning for $R$ will be blocked.

In the proposed $B M A-R S A$ algorithm, we provide migration-aware dynamic connection provisioning, based on the probabilistic migration label model before the migration begins, which can effectively avoid a large amount of data loss due to node upgrade. Compared with the method of re-establishing the connection after data transmission is interrupted, the proposed $B M A-R S A$ algorithm that provides pre-consideration protection for the service has great performance in terms of avoiding service interruption. During the process of label setting, the label of each node should be maintained. We can establish the path from the source to a selected node based on the node's final label for data transmission. It should be noted that the parameters $\alpha$ and $\beta$ are the weight coefficient for the link distance from the source to the selected node and the node upgrade probability. The value of $\alpha$ and $\beta$ can be modified at step 5, step 14 and step 15 for routing strategies which has various weighted label. For example, the $B M A-R S A$ algorithm will evolve to be the shortest path routing algorithm if we set $\alpha=1, \beta=0$. 
Algorithm 1 Brown-field Migration aware routing and spectrum assignment $(B M A-R S A)$ algorithm

Input: $G(V, E, P)$, NodeSet $=\{\operatorname{src}\}$, LinkSet $=\{\varnothing\}, R(S, D, B), V_{F I}, V_{F L}$

Output: Migration - aware path from src to node i, $F^{n, j t h}, S_{F L} / S_{F I}$

1 for each $R(S, D, B)$

$2 \quad \operatorname{Label}(\operatorname{src})=0, \operatorname{Label}(\mathrm{j})=\infty$;

3 for each node $j \in V-\{s r c\}$

$4 \quad \mid$ if node $j$ is neighbour of $\operatorname{src}$ then

5

6

7 


\section{Performance Evaluation and Analysis}

The performance of the proposed BMA-RSA algorithm is evaluated using distanceadaptive modulation format selection on USNET topology (24 nodes and 43 links) and NSFNET topology (14 nodes and 21 links), as shown in Figure 3a,b. The distance and spectrum occupation for various bit rates are shown in Table 2. We used JAVA to set up a simulation platform to solve the situation. The simulation requires a total of $8 \mathrm{THz}$ spectra for each link, which is evenly divided in each direction. A total of 500,000 connection requests following Poisson arrival and exponential departure were generated in each simulation run, and the bitrate demands of the connection requests are chosen uniformly among $\{40,100,200,400\}$ Gbps. For each traffic load scenario, 20 simulation experiments were conducted for different serials of connection requests. Both the mean and confidence interval at the $95 \%$ were evaluated and plotted as "I" in the result graphs. Spectrum continuity and contiguity are taken into consideration. During the process of node upgrade, we assumed that no new services were accommodated for in the network. Therefore, when the network node is selected to be upgraded, we changed the node attributes from the fixed-grid node to the flex-grid node. In our simulation, we generated a large number of services according to the traditional model, and analyzed the traffic information of each node statistically as the basis for analyzing and computing the upgrade probability of nodes. Since traffic may influence the migration strategies, two traffic models, in which traffic either uniformly or non-uniformly distributed among all the nodes are considered in practical fixed/flex-grid optical networks. In the non-uniform case, traffic is distributed according to the population of the city at the corresponding node and is positively correlated with the population [25]. In the uniform case, traffic is evenly distributed at all nodes. For the traffic models, the upgrading of 8 nodes and 16 nodes is considered, respectively. For each request, in order to verify the performance of the brown-field migration aware routing and spectrum assignment algorithm, we use traditional K-shortest-path routing and spectrum assignment $(K-S P-R S A)$ algorithm with first fit spectrum assignment $(k=1,2,3)$ as a comparison. In this section, we focused on the terms of bandwidth blocking ratio, which represents the rejected request bandwidth over the total bandwidth, and connection interrupted ratio, which is defined by the number of services interrupted due to node upgrade over the total number of deployed service connection, the result is statistically averaged after multiple simulations. The simulations run on a computer with $1.8 \mathrm{GHz}$ Intel Core i7-8565U CPU. The IntelliJ IDEA 2020 is installed in this computer to implement the proposed algorithm.

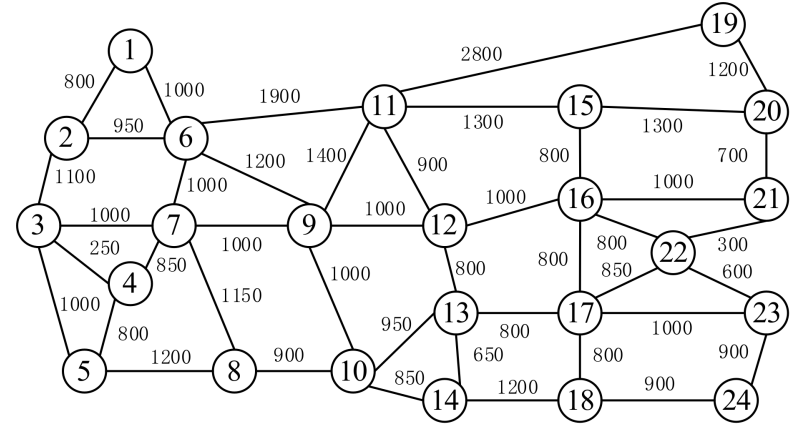

(a)

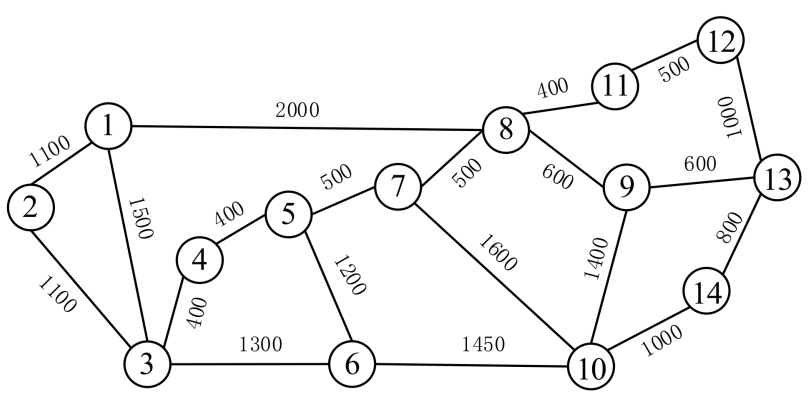

(b)

Figure 3. Simulation topology. (a) USNET topology (24 nodes and 43 links) (b) NSFNET topology (14 nodes and 21 links). 
Table 2. Distance and Spectrum Occupation for Various Bit Rates in Flex-Grid.

\begin{tabular}{|c|c|c|c|c|}
\hline Traffic Demands (Gb/s) & Modulation Format & Operating Bandwidth (GHz) & Distance (km) & \# Slots \\
\hline \multirow{3}{*}{40} & BPSK & 50 & 6000 & 4 \\
\hline & QPSK & 25 & 3000 & 2 \\
\hline & 8QAM & 25 & 1000 & 1 \\
\hline \multirow{5}{*}{100} & BPSK & 75 & 4500 & 6 \\
\hline & QPSK & 50 & 3500 & 4 \\
\hline & QPSK & 37.5 & 3000 & 3 \\
\hline & 8QAM & 25 & 2500 & 2 \\
\hline & 16QAM & 25 & 1500 & 2 \\
\hline \multirow{5}{*}{200} & BPSK & 100 & 2500 & 8 \\
\hline & QPSK & 75 & 1500 & 6 \\
\hline & 8QAM & 62.5 & 1000 & 5 \\
\hline & 16QAM & 43.75 & 700 & 4 \\
\hline & 32QAM & 37.5 & 500 & 3 \\
\hline \multirow{5}{*}{400} & BPSK & 200 & 2000 & 16 \\
\hline & QPSK & 150 & 1000 & 12 \\
\hline & 8QAM & 100 & 800 & 8 \\
\hline & 16QAM & 75 & 600 & 6 \\
\hline & 32QAM & 56.25 & 200 & 5 \\
\hline
\end{tabular}

Comparison of the proposed $B M A-R S A$ algorithm and SP-RSA algorithm, by upgrading 8 nodes in USNET topology and upgrading 5 nodes in NSFNET topology under a uniform and non-uniform traffic model in terms of BBR, are shown in Figure 4. We set $\alpha=1, \beta=2$ and $\alpha=1, \beta=0$ for their topologies, respectively. We can see that the trend of the bandwidth blocking ratio versus traffic load in USNET topology and NSFNET topology is similar. It is obvious that the BBR increases with the increase of the traffic load in both algorithms in two topologies. Meanwhile, as the number of paths (i.e., the value of k) selected increases, the bandwidth blocking ratio of both algorithms decreases. The reason is that a larger number of short paths mean more options for data transmission, which will more effectively resist service interruption. Meanwhile, we find that the BBR under the NSFNET topology is generally higher than that under the USNET topology. This is because USNET has a higher average link connectivity compared with NSFNET, and more service carrying paths based on migration awareness can be provided, which can reduce BBR.

Figure 5 shows that the bandwidth blocking ratio varies with different traffic load under different traffic models in USNET topology by updating 16 nodes and NSFNET topology updating 10 nodes. We can see that the trend of the bandwidth blocking ratio versus traffic load in USNET topology and NSFNET topology is similar. We can find that $B M A-R S A$ algorithm has a comparable performance with the $S P$-RSA algorithm in terms of bandwidth blocking ratio. For example, in Figure $5 a$, at 620 Erlang traffic load, compared with the SP-RSA algorithm, the bandwidth blocking ratio of the proposed $B M A-R S A$ algorithm has increased by $0 \%, 0 \%, 0.0012 \%$, respectively, corresponding to $\mathrm{K}=1, \mathrm{~K}=2$, $\mathrm{K}=3$. Likewise, at 780 Erlang traffic load shown in Figure $5 \mathrm{~b}$, the bandwidth blocking ratio of the BMA-RSA algorithm has increased by $0.064 \%, 0.084 \%, 0.064 \%$ compared with the SP-RSA algorithm. This is because the traditional SP-RSA algorithm establishes the shortest path without considering the probability of node upgrade, and the shorter path means a lower bandwidth blocking ratio. However, the proposed BMA-RSA algorithm fully considers the impact of node upgrade, in order to minimize the impact of a network upgrade on the services that have been carried, it may choose a relatively long path which 
may not the optimal one. As a result, spectrum resources are not utilized optimally, which inevitably increases the bandwidth blocking ratio. The strategy under the uniform traffic model achieves better performance compared with the non-uniform traffic model. This is because that in the non-uniform case, traffic is unevenly distributed, which leads to a lower bandwidth blocking ratio. By comparing Figures 4 and 5, we reach the conclusion that upgrading 16 nodes and upgrading 10 nodes from fixed-grid to flex-grid nodes can lead to a lower bandwidth blocking ratio than can be achieved by upgrading 8 nodes and upgrading 5 nodes in USNET and NSFNET, respectively. Because the super optical channel can only establish connections between flex-grid nodes, upgrading more nodes means more flexibility in the network.

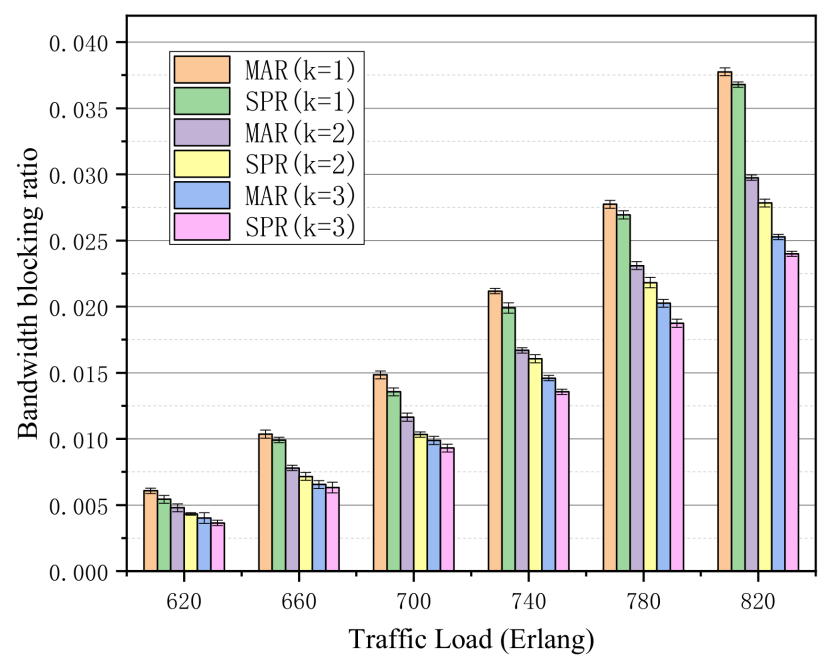

(a)

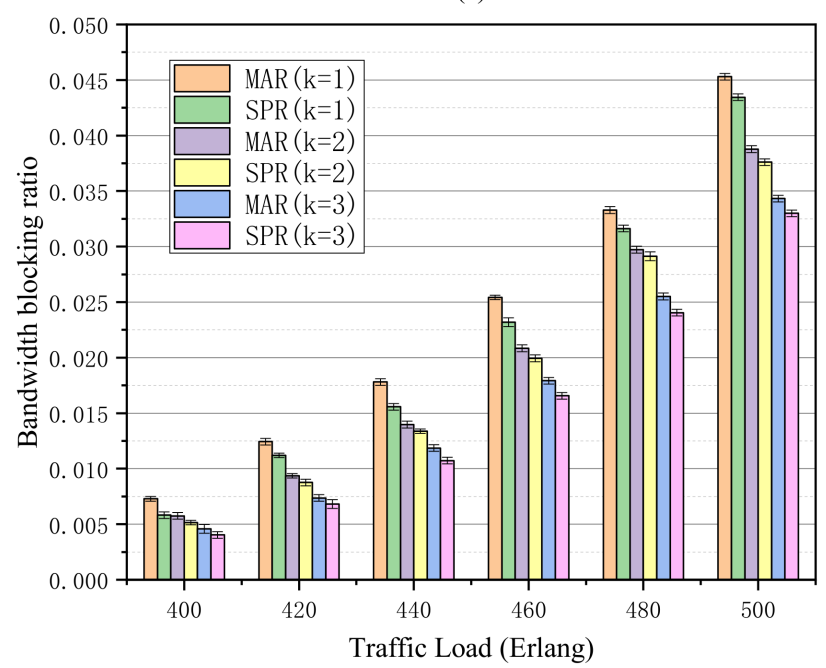

(c)

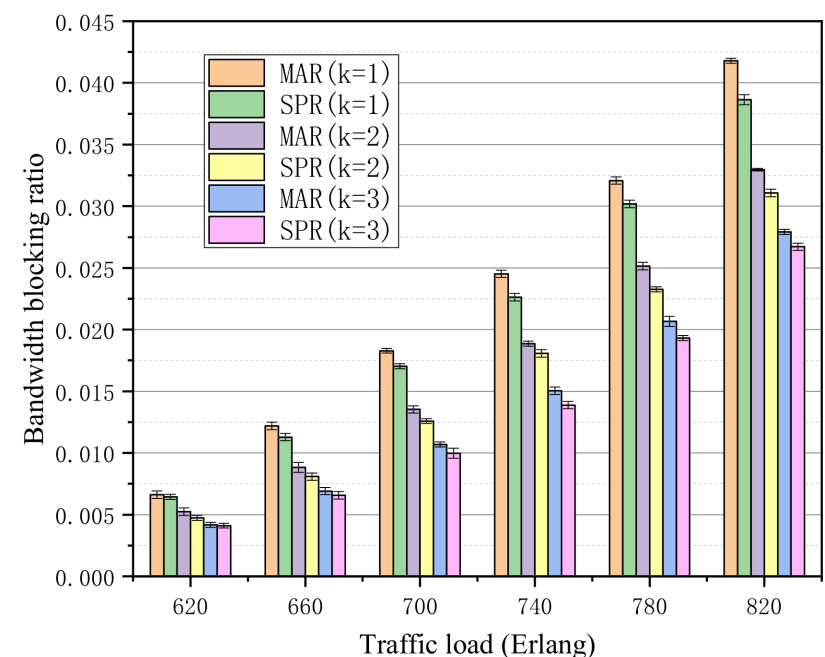

(b)

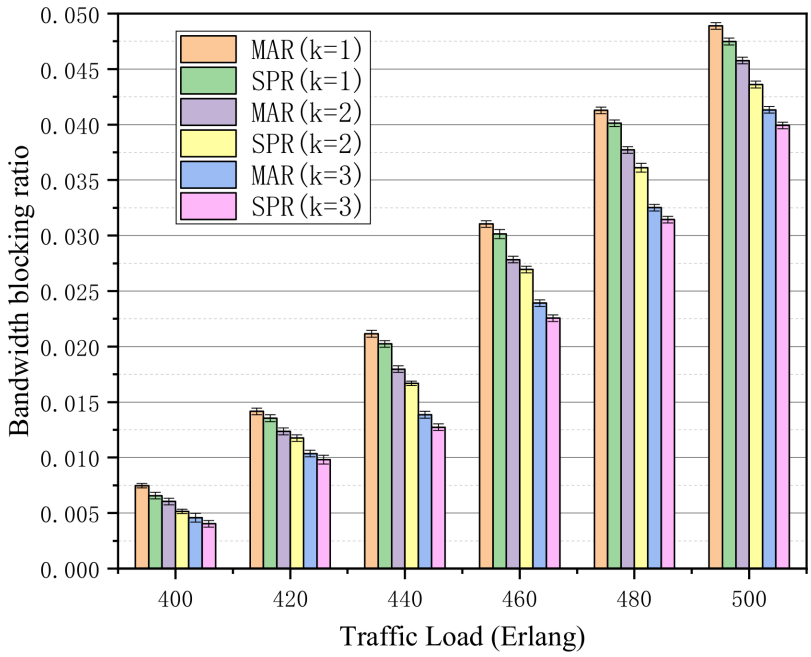

(d)

Figure 4. Simulation and results. (a) BBR under uniform traffic model when 8 nodes are upgraded in USNET; (b) BBR under non-uniform traffic model when 8 nodes are upgraded in USNET; (c) BBR under uniform traffic model when 5 nodes are upgraded in NSFNET; (d) BBR under non-uniform traffic model when 5 nodes are upgraded in NSFNET. 


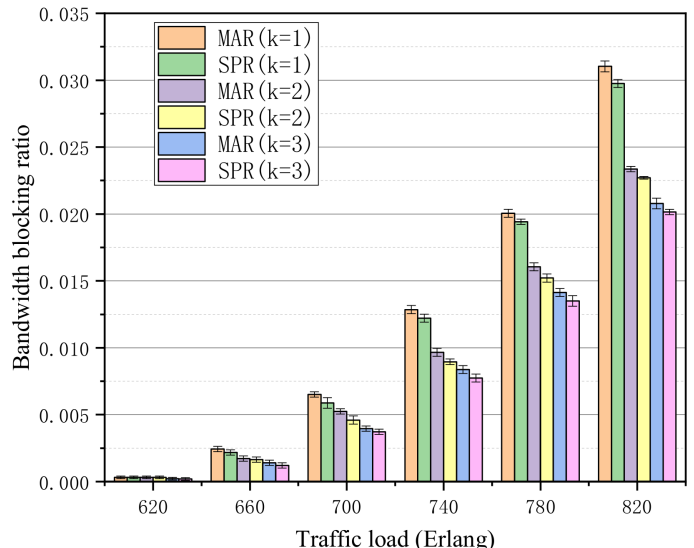

(a)

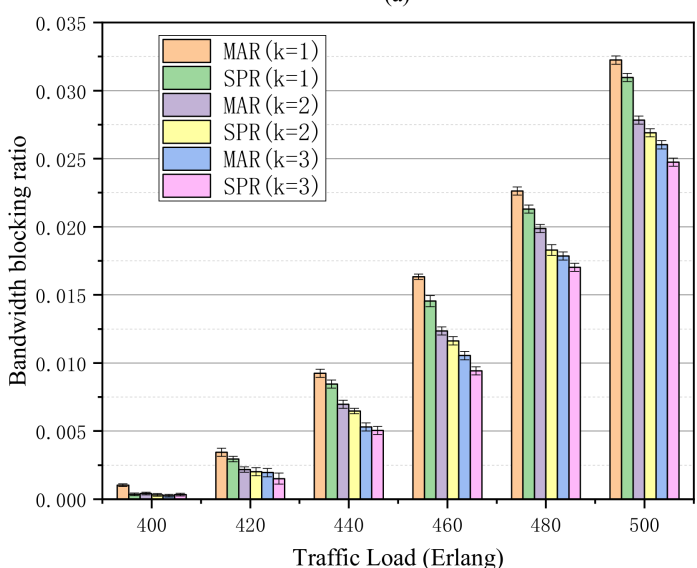

(c)

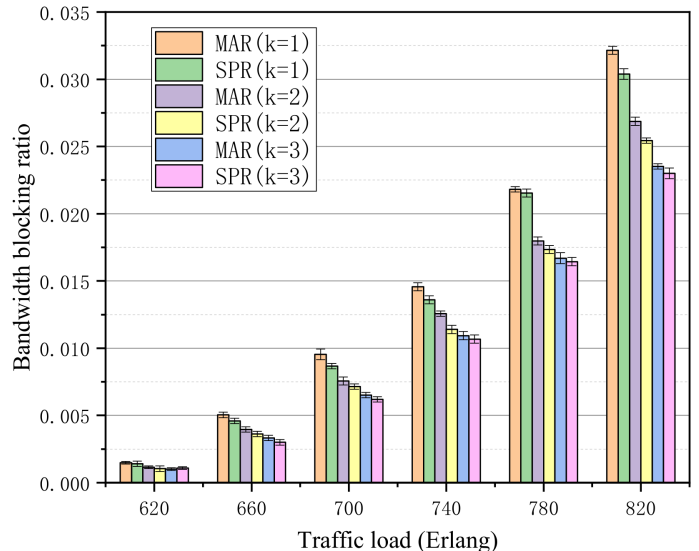

(b)

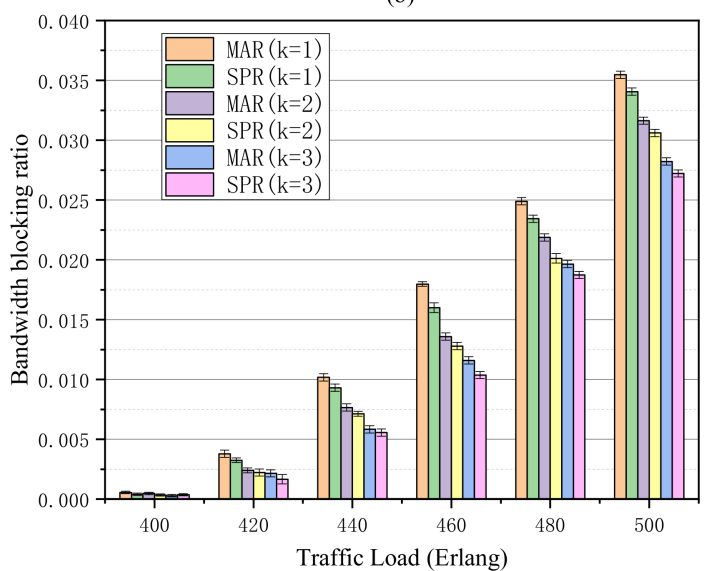

(d)

Figure 5. Simulation and results. (a) BBR under uniform traffic model when 16 nodes are upgraded in USNET; (b) BBR under non-uniform traffic model when 16 nodes are upgraded in USNET; (c) BBR under uniform traffic model when 10 nodes are upgraded in NSFNET; (d) BBR under non-uniform traffic model when 10 nodes are upgraded in NSFNET.

Figures 6 and 7 show the connection interruption ratio for different traffic loads under uniform and non-uniform traffic models in the network of the USNET topology with 8 nodes or 16 nodes upgraded and the NSFNET topology with 5 nodes or 10 nodes upgraded. During the simulation process, we set $k=2$ as an example to verify the performance of different $\beta$ on connection interruption ratio under the same $\alpha$ value. We can see that when the value of alpha is fixed, as the value of beta increases, the CIR gradually decreases. This is because node-upgrade probability is an important factor that influences the connection interruption of the service supply. By providing a higher weight to the upgrade probability, the risk of service interruption and traffic loss caused by node upgrade can be effectively reduced. Apparently, the proposed BMA-RSA algorithm achieves considerable benefits in connection interruption ratio. For example, as shown in Figure 7a, at 620 Erlang traffic load, $B M A-R S A$ algorithm which sets the weight coefficient of node upgrade probability to 2,5 , and 10 , respectively, decreases the connection interruption ratio by $0.38 \%, 0.73 \%$, and $1.12 \%$ compared with the $S P-R S A$ algorithm which sets the weight coefficient of node upgrade probability as 0 . Meanwhile, the connection interruption ratio decreases as the value of parameter $\beta$ increases. This can be explained as follows: in fixed/flex-grid optical networks, node update has a huge influence on the performance of data transmission. The traditional algorithm does not consider the impact of node upgrade, in the process of data transmission, when a node on the path carrying the traffic upgrades, the data transmission is interrupted, resulting in data loss, thus leading to a high connection interruption ratio. Meanwhile, the connection interruption ratio under the non-uniform traffic model decreases faster than that under uniform traffic model. This is because, under 
the non-uniform traffic model in which traffic is unevenly distributed, some nodes have relatively high probability are easier to be upgraded to flex-grid, causing a large number of data loss. The proposed algorithm can effectively avoid this situation.

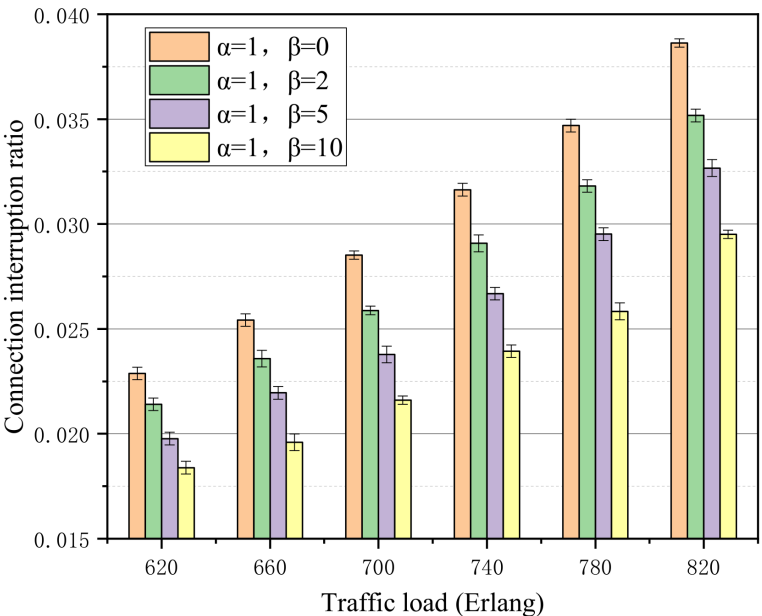

(a)

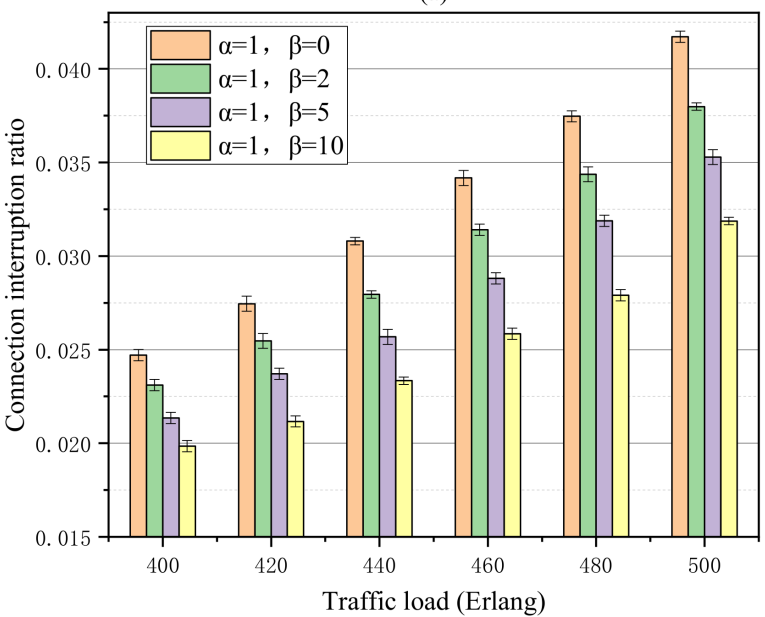

(c)

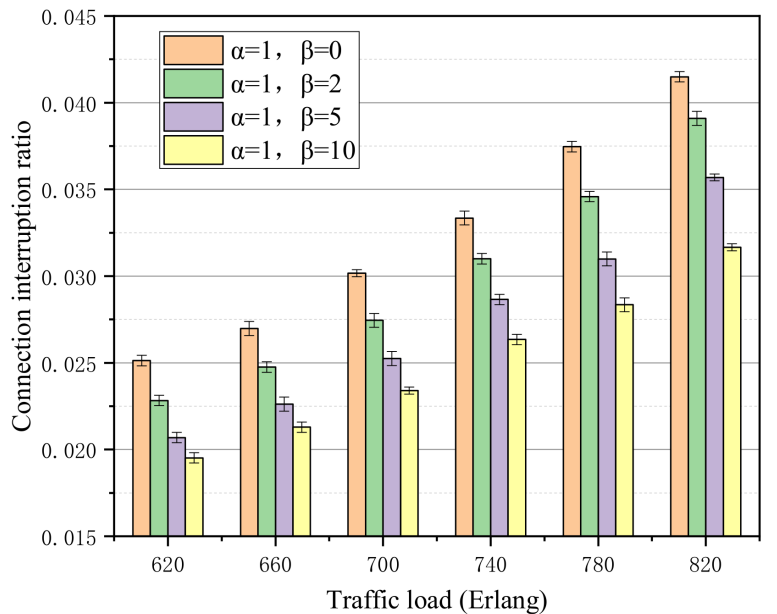

(b)

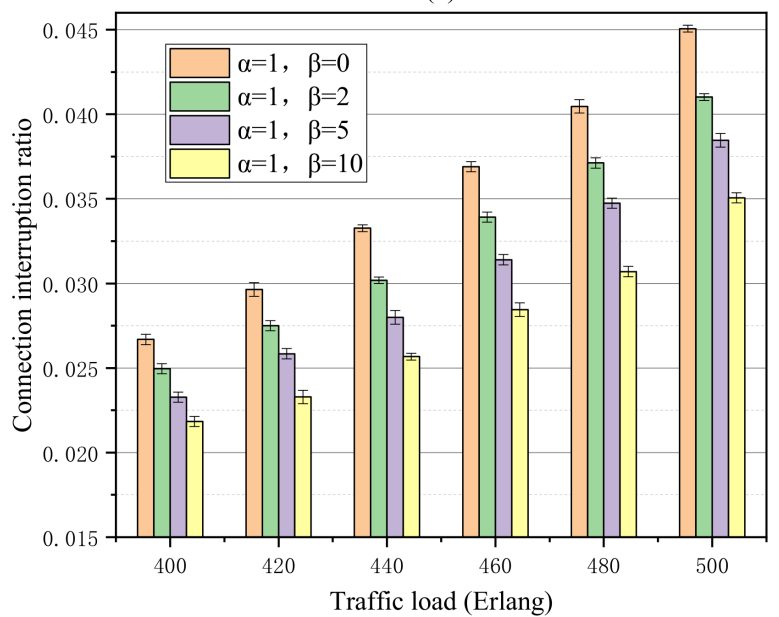

(d)

Figure 6. (a) CIR under uniform traffic model when 8 nodes are upgraded in USNET; (b) CIR under non-uniform traffic model when 8 nodes are upgraded in USNET; (c) CIR under uniform traffic model when 5 nodes are upgraded in NSFNET; (d) CIR under non-uniform traffic model when 5 nodes are upgraded in NSFNET.

Figure 7a-d illustrates the connection interruption ratio versus different traffic loads in USNET with 16 upgraded nodes and NSFNET with 10 upgraded nodes. Similarly, in Figure $7 b$, at 780 Erlang traffic load, the $B M A-R S A$ algorithm, which sets the node upgrade probability weight coefficient as $2,5,10$, creates the gap of $0.43 \%, 0.93 \%$, and $1.23 \%$ lower than the SP-RSA algorithm, which sets the weight coefficient of node upgrade probability as 0 , respectively. Meanwhile, under the same traffic load, the network with 16 upgraded nodes achieves a higher connection interruption ratio compared with the network with 8 upgraded nodes. We obtain a similar conclusion under the NSFNET topology. This can be explained that much more upgraded nodes (i.e., $50 \%$ of the nodes in the network) will promote more interruptions caused by nodes. For example, at the 820 Erlang traffic load under the non-traffic model, the connection interruption ratio is as high as $8.43 \%$. Through the analysis of the simulation results, we can conclude that the proposed $B M A-$ $R S A$ algorithm has great advantages. This is because it can greatly reduce the connection interruption ratio while increasing the extremely small bandwidth blocking ratio, which can be almost negligible. Additionally, by taking the node probability into consideration, brown- 
field migration aware routing and spectrum assignment (BMA-RSA) algorithm we proposed can achieve migration-aware dynamic connection provisioning before network migration occurs, which can greatly improve the overall network performance because during the brown-field migration process, node upgrade probability is an important factor affecting service connection. In order to provide service reliability guarantee, we must provide it with more attention.

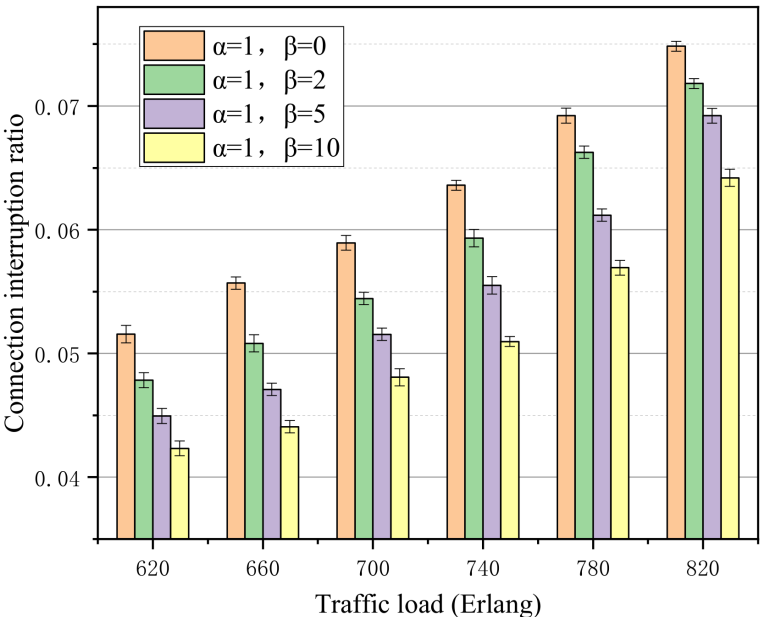

(a)

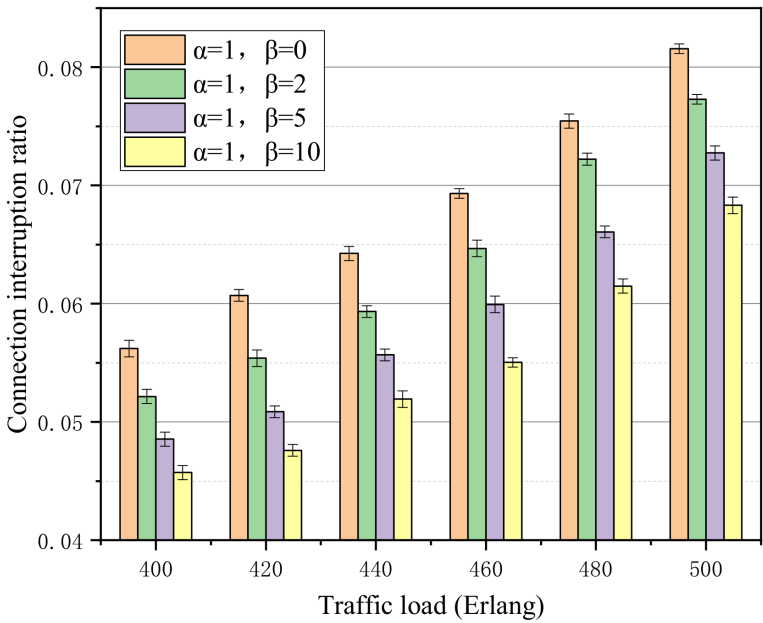

(c)

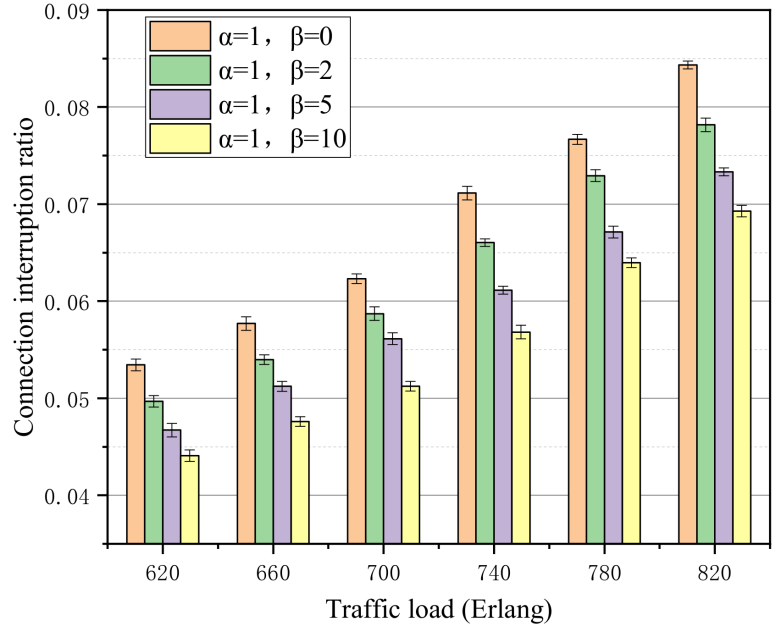

(b)

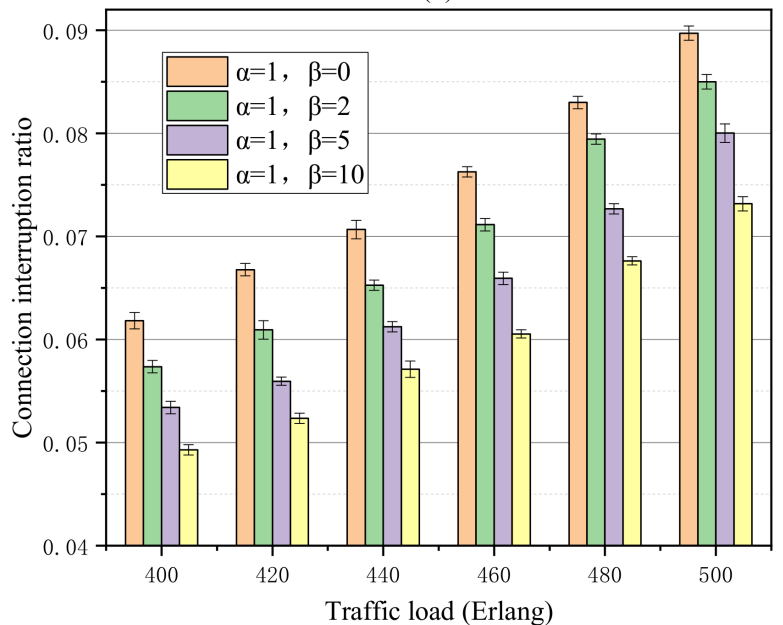

(d)

Figure 7. (a) CIR under uniform traffic model when 16 nodes are upgraded in USNET; (b) CIR under non-uniform traffic model when 16 nodes are upgraded in USNET; (c) CIR under uniform traffic model when 10 nodes are upgraded in NSFNET; (d) CIR under non-uniform traffic model when 10 nodes are upgraded in NSFNET.

\section{Conclusions}

During the migration from fixed-grid optical networks to flex-grid networks, fixed / flexgrid optical networks will appear as a transition. Under this situation, migration-aware dynamic connection provisioning to avoid data transmission interruption, which can be caused by node upgrades before the migration begins, needs to be solved. We propose a probabilistic migration-label model-based brown-field migration aware routing and spectrum assignment algorithm. By taking the node upgrade probability into consideration, the algorithm proposed in this paper has better performance. Furthermore, given that the bandwidth blocking ratio performance is not improved in our algorithm, methods to improve our strategy to further reduce the bandwidth blocking ratio of a network, while reducing the connection interruption ratio, will be the basis for our future research. Furthermore, in our future work, we will consider the planning problem using ILP solu- 
tions in static scenarios and study the deployment of fixed/flex-grid optical networks to achieve corresponding results. Additionally, how to jointly consider load balancing into migration-aware routing and spectrum assignment proves to be a very interesting topic.

Author Contributions: Conceptualization, X.Y., J.W. and Y.Z.; methodology, X.Y. and Y.Z.; software, K.Z. and J.L.; validation, K.G. and X.G.; writing-original draft preparation, X.Y.; writing-review and editing, J.W., K.Z., J.L., Y.Z., K.G., X.G. and J.Z.; supervision, J.Z. All authors have read and agreed to the published version of the manuscript.

Funding: This paper was supported by the science and technology project from State Grid Corporation of China: Research on Key Technologies of 100G Large Capacity Backbone Optical Transmission Network (SGXJDK00DJJS2000101).

Institutional Review Board Statement: Not applicable.

Informed Consent Statement: Not applicable.

Data Availability Statement: The data presented in this study are available on request from the corresponding author. The data are not publicly available due to privacy.

Conflicts of Interest: The authors declare no conflict of interest.

\section{References}

1. Emara, T.Z.; Huang, J.Z. Distributed Data Strategies to Support Large-Scale Data Analysis Across Geo-Distributed Data Centers. IEEE Access 2020, 8, 178526-178538. [CrossRef]

2. Balanici, M.; Pachnicke, S. Hybrid electro-optical intra-data center networks tailored for different traffic classes. IEEE/OSA J. Opt. Commun. Netw. 2018, 10, 889-901. [CrossRef]

3. Wei, W.; Gu, H.; Wang, K.; Yu, X.; Liu, X. Improving Cloud-Based IoT Services Through Virtual Network Embedding in Elastic Optical Inter-DC Networks. IEEE Internet Things J. 2019, 6, 986-996. [CrossRef]

4. Gerstel, O.; Jinno, M.; Lord, A.; Yoo, S.J.B. Elastic optical networking: A new dawn for the optical layer? IEEE Commun. Mag. 2012, 50, s12-s20. [CrossRef]

5. Gringeri, S.; Basch, B.; Shukla, V.; Egorov, R.; Xia, T.J. Flexible architectures for optical transport nodes and networks. IEEE Commun. Mag. 2010, 48, 40-50. [CrossRef]

6. Zhang, G.; de Leenheer, M.; Morea, A.; Mukherjee, B. A survey on OFDM-based elastic core optical networking. IEEE Commun. Surv. Tut. 2013, 15, 65-87. [CrossRef]

7. Zhu, Z.; Lu, W.; Zhang, L.; Nirwan, A. Dynamic service provisioning in elastic optical networks with hybrid single-/multi-path routing. J. Lightw. Technol. 2013, 31, 15-22. [CrossRef]

8. Xu, Z.; Tremblay, C.; Archambault, É.; Furdek, M.; Chen, J.; Wosinska, L.; Bélanger, M.P.; Littlewood, P. Flexible Bandwidth Allocation in Filterless Optical Networks. IEEE Commun. Lett. 2015, 19, 565-567. [CrossRef]

9. ITU-T SG-15. Spectrum Grids for WDM Applications: DWDM Frequency Grid; ITU-T G.694.1: Geneva, Switzerland, 2012.

10. Palkopoulou, E.; Angelou, M.; Klonidis, D.; Christodoulopoulos, K. Quantifying spectrum, cost, and energy efficiency in migrationed-grid and flex-grid networks. J. Opt. Commun. Netw. 2012, 4, B42-B51. [CrossRef]

11. Sheryl, L.; Mark, D.F. Benefits and Requirements of Flexible-Grid ROADMs and Networks. J. Opt. Commun. Netw. 2013, 5, A19-A27.

12. Masahiko, J.; Hidehiko, T.; Bartlomiej, K. Spectrum-Efficient and Scalable Elastic Optical Path Network: Architecture, Benefits, and Enabling Technologies. IEEE Commun. Mag. 2009, 47, 66-73.

13. Masahiko, J.; Hidehiko, T.; Yoshiaki, S. Multiflow Optical Transponder for Efficient Multilayer Optical Networking. IEEE Commun. Mag. 2012, 50, 56-65.

14. Khodashenas, P.E. Comparison of Spectral and Spatial Super-Channel Allocation Schemes for SDM Networks. J. Lightwave Technol. 2016, 34, 2710-2716. [CrossRef]

15. Velasco, L.; Wright, P.; Lord, A.; Junyent, G. Saving CAPEX by Extending Flexgrid-Based Core Optical Networks toward the Edges. IEEE/OSA J. Opt. Commun. Netw. 2013, 5, A171-A183. [CrossRef]

16. Lechowicz, P.; Perello, J.; Spadaro, S.; Walkowiak, K. Brown-field Gradual Migration Planning Toward Spectrally-Spatially Flexible Optical Networks. In Proceedings of the 2020 International Conference on Optical Network Design and Modeling (ONDM), Castelldefels, Barcelona, Spain, 18-21 May 2020.

17. Ahmed, T.; Rahman, S.; Tornatore, M.; Mukherjee, B. Dynamic Routing and Spectrum Assignment in Co-Existing Fixed/Flex-Grid Optical Networks. In Proceedings of the IEEE International Conference on Advanced Networks and Telecommunications Systems (ANTS), Indore, India, 16-19 December 2018.

18. Zhang, Y.; Zhang, Y.; Bose, S.K.; Shen, G. Migration from Fixed to Flexible Grid Optical Networks with Sub-Band Virtual Concatenation. J. Light. Technol. 2017, 35, 1752-1765. [CrossRef] 
19. Ahmed, T.; Rahman, S.; Ferdousi, S.; Tornatore, M.; Mitra, B.C.; Mukherjee, B. Dynamic routing, spectrum, and modulation-format allocation in mixed-grid optical networks. J. Opt. Commun. Netw. 2020, 12, 79-88. [CrossRef]

20. Rosanna, P.; Gabriella, B.; Stefano, P.; Fabrizio, F. Networking Planning Strategies for Next-Generation Flexible Optical Networks. J. Opt. Commun. Netw. 2015, 7, A511-A525.

21. Ruiz, M.; Velasco, L.; Lord, A.; Fonseca, D.; Pioro, M.; Wessaly, R.; Fernandez-Palacios, J.P. Planning fixed to flexgrid gradual migration: Drivers and open issues. IEEE Commun. Mag. 2014, 52, 70-76. [CrossRef]

22. Christodoulopoulos, K.; Soumplis, P.; Varvarigos, E. Planning Flexible Optical Networks under Physical Layer Constraints. J. Opt. Commun. Netw. 2013, 5, 1296-1312. [CrossRef]

23. Zhu, Q.; Yu, X.; Zhao, Y.; Nag, A.; Zhang, J. Auxiliary-Graph-based Energy-Efficient Traffic Grooming in IP-over-Fixed/Flex-Grid Optical Networks. J. Lightwave Technol. 2021, 11, 3057389. [CrossRef]

24. Yu, X.; Tornatore, M.; Zhao, Y.; Biswanath, M. When and how should the optical network be upgraded to flex grid? In Proceedings of the European Conference and Exhibition on Optical Communication (ECOC), Cannes, France, 24 November 2014.

25. Yu, X.; Tornatore, M.; Xia, M.; Wang, J.; Zhang, J.; Zhao, Y.; Zhang, J.; Mukherjee, B. Migration from fixed grid to flexible grid in optical network. IEEE Commun. Mag. 2015, 53, 34-43. [CrossRef]

26. Yu, X.; Tornatore, M.; Xia, M.; Zhao, Y.; Zhang, J.; Mukherjee, B. Brown-field migration from fixed grid to flexible grid in optical networks. In Proceedings of the Optical Fiber Communications Conference (OFC), Los Angeles, CA, USA, $22-26$ March 2015.

27. Yu, X.; Zhao, Y.; Chen, B.; Zhang, J.; Li, Y.; Zhang, G.; Chen, X.; Zhang, J. Migration-Aware Dynamic Connection Provisioning in Optical Networks Evolving from Fixed Grid to Flexible Grid. In Proceedings of the 2016 Asia Communications and Photonics Conference (ACP), Wuhan, China, 2-5 November 2016.

28. Ramamurthy, S.; Laxman, S.; Biswanath, M. Survivable WDM mesh networks. J. Lightw. Technol. 2003, 21, 870-883. [CrossRef]

29. Zhou, D.; Subramaniam, S. Survivability in optical networks. IEEE Netw. 2000, 14, 16-23.

30. Wang, W.; Doucette, J. Availability optimization in shared-backup path protected networks. IEEE/OSA J. Opt. Commun. Netw. 2018, 10, 451-460. [CrossRef]

31. Wang, C.; Shen, G.; Bose, S.K. Distance Adaptive Dynamic Routing and Spectrum Allocation in Elastic Optical Networks with Shared Backup Path Protection. J. Lightwave Technol. 2015, 33, 955-2964. [CrossRef]

32. Zhu, R.; Li, S.; Wang, P.; Tan, Y.; Yuan, J. Gradual Migration of Co- Existing Fixed/Flexible Optical Networks for Cloud-Fog Computing. IEEE Access 2020, 8, 50637-50647. [CrossRef] 